\title{
MiR-539-5p alleviates sepsis-induced acute lung injury by targeting ROCK1
}

\author{
Li Meng $^{1 *}$, Haohao Cao ${ }^{2 *}$, Chunhua Wan${ }^{1}$, Lintao Jiang ${ }^{3}$ \\ ${ }^{1}$ Departement of Anesthesiology, The Affiliated Hospital of Hubei Provincial Government, Wuhan, China \\ ${ }^{2}$ Departement of Critical Care Medicine, Wuhan Forth Hospital, Wuhan, Hubei Province, 430000, \\ China \\ ${ }^{3}$ Departement of Emergency, Central Hospital of Wuhan, Wuhan, Hubei Province, 430000, China \\ *both authors equally contributed to this paper
}

\begin{abstract}
Introduction. Sepsis-induced acute lung injury (ALI) is an inflammatory process involved with simultaneous production of inflammatory cytokines and chemokines. In this study, we investigated the regulatory role of miR-539-5p in sepsis-induced ALI using a mouse model of cecal ligation puncture (CLP) and an in vitro model of primary murine pulmonary microvascular endothelial cells (MPVECs).

Material and methods. Adult male C57BL/6 mice were intravenously injected with or without miR-539-5p agomir or scrambled control one week before CLP operation. MPVECs were transfected with miR-539-5p mimics or control mimics, followed by lipopolysaccharide (LPS) stimulation. ROCK1 was predicted and confirmed as a direct target of miR-539-5p using dual-luciferase reporter assay. In rescue experiment, MPVECs were co-transfected with lentiviral vector expressing ROCK1 (or empty vector) and miR-539-5p mimics $24 \mathrm{~h}$ before LPS treatment. The transcriptional activity of caspase-3, the apoptosis ratio, the levels of miR-539-5p, interleukin- $1 \beta$ (IL- $1 \beta$ ), interleukin-6 (IL-6), and ROCK1 were assessed.

Results. Compared to sham group, mice following CLP showed pulmonary morphological abnormalities, elevated production of IL- $1 \beta$ and IL-6, and increased caspase- 3 activity and apoptosis ratio in the lung. In MPVECs, LPS stimulation resulted in a significant induction of inflammatory cytokine levels and apoptosis compared to untreated cells. The overexpression of miR-539-5p in septic mice alleviated sepsis-induced pulmonary injury, apoptosis, and inflammation. MiR-539-5p also demonstrated anti-apoptotic and anti-inflammatory effect in LPS-treated MPVECs. The upregulation of ROCK1 in MPVECs recovered miR-539-5p-suppressed caspase-3 activity and proinflammatory cytokine production.

Conclusion. In conclusion, miR-539-5p alleviated sepsis-induced ALI via suppressing its downstream target ROCK1, suggesting a therapeutic potential of miR-539-5p for the management of sepsis-induced ALI. (Folia Histochemica et Cytobiologica 2019, Vol. 57, No. 4, 168-178)
\end{abstract}

Key words: mouse; acute lung injury; sepsis; inflammation; MPVEC cells; miRNA; ROCK1; caspase-3; cytokines

Correspondence address: Chunhua Wan

Department of Anesthesiology, The Central Hospital of Wuhan, Unit 2, Building 23, Phase II, Yuejiazui Staff Residential

Quarter, No. 46, Joy Avenue,

Hongyuan District, Wuhan, Hubei, China

phone: $86+0278-2211435$

e-mail: ChunhuaWanrui@163.com

\section{Introduction}

Sepsis is life-threatening syndrome characterized by an excessive systemic inflammatory response to infections induced by pathogenic bacteria, fungi, or viruses [1]. Patients with severe sepsis commonly present with microvascular thrombosis and subsequent multiple organ dysfunction [2]. Acute lung injury (ALI) 
is a clinical syndrome consisting of a wide range of acute hypoxemic respiratory failure disorders and has become one of the most common complications of severe sepsis [3]. Pathophysiological studies revealed that the injury to the alveolar epithelium and pulmonary endothelial cells leads to an induction of epithelial permeability, pulmonary edema, and acute respiratory failure during the development of ALI [4]. However, no current available pharmacologic therapy has been recommended as a standard management of ALI [5].

Sepsis-induced ALI is an acute inflammatory process involved with simultaneous production of inflammatory cytokines and chemokines. Interleukin- $1 \beta$ (IL- $1 \beta)$, for example, has been found in bronchoalveolar lavage fluids from ALI patients [6]. In patients with persistent acute respiratory distress syndrome, a more severe form of ALI, the high ratio of IL- $1 \beta$ to its antagonist suggested an essential role of IL- $1 \beta$ in maintaining chronic inflammatory condition in lungs [7]. The other key step in ALI progression is the extensive apoptosis of alveolar epithelial cells, which has been considered as a primary cause of lung epithelium death in ALI patients [8]. Studies in murine endotoxin-induced lung injury models showed that the accumulation of soluble Fas ligand in Fas-dependent pathway induced apoptosis in lung epithelial cells [9, 10]. Thus, the blockage of inflammatory response and apoptotic signaling in the lung might be a useful therapeutic strategy in ameliorating sepsis-induced ALI.

MicroRNAs (miRNAs) are a class of small non-coding RNAs that have shown an emerging regulatory role in many pathological processes, including sepsis-induced organ dysfunction [11]. The overexpression of miR-146a attenuated sepsis-triggered myocardial dysfunction in vitro via inhibiting NF- $\kappa \mathrm{B}$ activation and the expression of inflammatory cytokines [12]. MiR-27a mediated the protective effect of paclitaxel in the damaged liver from septic mice by suppressing NF- $\kappa \mathrm{B} / \mathrm{TAB} 3$ signaling pathway [13]. MiR-539-5p has been shown as a potent regulator in choroidal neovascularization and the migration of mesenchymal stem cells in fracture healing $[14,15]$. However, whether it may exert beneficial effect in ALI remains unclear.

In this study, we aimed to investigate the regulatory role and potential molecular mechanism of miR-539-5p in sepsis-induced ALI by using a murine sepsis model and the primary murine pulmonary microvascular endothelial cells. The degree of lung tissue injury, the level of apoptosis and the expression of inflammatory cytokines were examined. Our findings may suggest a novel therapeutic approach in the management of septic ALI.

\section{Materials and methods}

Mouse model of sepsis. A total of 24 adult male C57BL/6 mice (28-32 g) were purchased from Charles River Laboratories China and maintained in a controlled environment (12 $\mathrm{h}$ alternating light-dark cycle, $22-24^{\circ} \mathrm{C}, 60 \%$ humidity) with ad libitum access to food and water. All experiments in this study were approved by the Animal Care and Use Committee of The Central Hospital of Wuhan, and performed following the Guide for the Care and Use of Laboratory Animals [16]. After one-week acclimatization, 18 mice underwent cecal ligation and puncture (CLP) surgery to establish the model of CLP-induced sepsis as previously described $[17,18]$. In brief, mice were anesthetized by i.p. injection of $10 \%$ chloral hydrate $(3 \mathrm{~mL} / \mathrm{kg}$, Sigma-Aldrich, St. Louis, MI, USA) and fixed on the operating table in supine position. A 4-mm longitudinal midline incision was made to expose the cecum. The exposed cecum was ligated at $10 \mathrm{~mm}$ from the tip using 3-0 silk sutures and then punctured once with a 20 -gauge needle at $5 \mathrm{~mm}$ distal from the ligation. After extruding a small amount of feces by gently squeezing the cecum, the bowel was repositioned and the abdominal musculature, peritoneum and skin were closed using sterile sutures. Immediately after the surgery, $5 \mathrm{~mL} / 100 \mathrm{~g}$ saline was subcutaneously injected for fluid resuscitation. Sham-operated mice $(n=6)$ underwent the same surgical procedure without the ligation or puncture of the cecum.

In vivo delivery of plasmid DNA. CLP mice were randomly divided into three groups: CLP, CLP + NC agomir, and CLP + miR-539-5p agomir. The miRNA reagents, miR539-5p agomir and scrambled control (NC agomir), were purchased from GenePharm (Shanghai, China) and mixed with linear polyethyleneimine (PEI) nanoparticles (Sigma-Aldrich) as previously described [19]. A total of $200 \mu \mathrm{L}$ mixture containing $5 \mathrm{nmol}$ miRNA (miR-539-5p agomir or NC agomir) was intravenously injected via the tail vein into designated group one week before the CLP operation. Animals were euthanized with carbon dioxide asphyxia $24 \mathrm{~h}$ post-surgery. Lungs were harvested and stored properly for further analyses.

Histological analysis. The same portion of the lung samples in mice were used for histopathological examination. Tissues were fixed in $4 \%$ paraformaldehyde, embedded in paraffin, cut into $5 \mu \mathrm{m}$ sections, and then stained with hematoxylin and eosin $(\mathrm{H} \& \mathrm{E})$. The degree of lung damage was estimated using lung injury scoring by an investigator blinded to the experiment as previously described [20]. Five randomly selected fields were scored per slide at magnification $400 \times$.

Cell culture and transfection. Primary murine pulmonary microvascular endothelial cells (MPVECs) were isolated 
and cultured in DMEM (Sigma-Aldrich) as previously described [21]. The miR-539-5p mimics and control mimics $(50 \mathrm{nM})$ were synthesized by GenePharm and transfected into MPVECs using Lipofectamine 2000 (Invitrogen, Carlsbad, CA, USA). Twenty-four hours after transfection, cells were stimulated with $1 \mathrm{mg} / \mathrm{mL}$ lipopolysaccharide (LPS, Sigma-Aldrich). The transcriptional activity of caspase-3 was examined $30 \mathrm{~min}$ thereafter. The levels of miR-539-5p, interleukin-1 $\beta$ (IL-1 $\beta$ ), interleukin-6 (IL-6), and ROCK1 were assessed $6 \mathrm{~h}$ following the LPS treatment. In rescue experiment, MPVECs were co-transfected with $1 \mu \mathrm{g}$ recombinant lentiviral vector expressing ROCK1 (or empty lentiviral vector) and $50 \mathrm{nM}$ miR-539-5p mimics $24 \mathrm{~h}$ prior to the treatment with LPS. MPVECs co-transfected with empty lentiviral vector and control mimics were used as a control group.

Dual-luciferase reporter assay. ROCK1 3'-UTR fragment containing the putative binding site of miR-539-5p was amplified and cloned into the downstream of luciferase gene in the pmirGlo vector (GenePharm). The mutant ROCK1 3'-UTR was used to construct ROCK1-MUT vector. HEK-293 cells were plated into 12-well plates (Thermo Fisher Scientific, Waltham, MA, USA) and co-transfected with miR-539-5p mimics (or NC mimics) and ROCK1-WT (or ROCK1-MUT) at 70-80\% confluency. The luciferase activities were assessed 2 days after the transfection using Dual-Luciferase Reporter Assay System (Promega Biotech Co., Madison, WI, USA).

Quantitative real-time PCR (qRT-PCR). Target miRNA was extracted from mice lung tissues and MPVECs using mirVana $^{\mathrm{TM}}$ miRNA Isolation Kit (Invitrogen). The reverse transcription of miR-539-5p was performed using All-inOne $^{\mathrm{TM}}$ miRNA RT-qPCR Detection Kit (GeneCopoeia Inc., Rockville, MD, USA). Total RNAs were isolated using Trizol LS (Invitrogen) and reverse transcribed to cDNA using the ReverTra Ace qPCR RT Kit (Toyobo, Osaka, Japan) according to the manufacturer's instructions. Target genes were amplified using 7300 Real-Time PCR System (Applied Biosystem, Foster City, CA, USA). The expression of miR-539-5p was normalized by U6, while $\beta$-actin was used as internal control for mRNA expression. The primers used in this study were: miR-539-5p: GGAGAAAUUAUCCUUGGUGUGU; U6 forward: CTCGCTTCGGCAGCACA, U6 reverse: AACGCTTCACGAATTTGCGT; IL- $1 \beta$ forward: CCAGGATGAGGACCCAAGCA, IL- $1 \beta$ reverse: TCCCGACCATTGCTGTTTCC; IL-6 forward: TAGCCGCCCCACACAGACAG, IL-6 reverse: GGCTGGCATTTGTGGTTGGG; ROCK1 forward: AAAGAAAGGATGGAG-GATGAAGT, ROCK1 reverse: TGTAACAACAGCCGCTTATTTG; $\beta$-actin forward: ATCACTGCCACCCAGAAGAC, $\beta$-actin reverse: TTTCTAGACGGCAGGTCAGG.
Enzyme-linked immunosorbent assay (ELISA). Total proteins extracted from mice lung tissues and MPVEC lysates were prepared. Concentrations of IL- $1 \beta$ and IL- 6 were measured using ELISA kits (R\&D Systems, Minneapolis, MN, USA) according to the manufacturer's protocol.

Transcriptional activity assay. The transcriptional activity of caspase-3 in mice lung tissues and MPVECs were evaluated using Caspase-3 Assay Kit (Abcam, Cambridge, UK) according to the manufacturer's protocol. Lung samples were homogenized in RIPA buffer with protease inhibitor (Bio-Rad Laboratories, Hercules, CA, USA). MPVECs were lysed in cell lysis buffer $30 \mathrm{~min}$ after LPS stimulation. The protein concentration in all samples were adjusted to $100-150 \mu \mathrm{g}$ protein per $50 \mu \mathrm{L}$ cell lysis buffer. The mixture of $50 \mu \mathrm{L} 2 \times$ Reaction Buffer and $10 \mathrm{mM}$ DTT were added to $50 \mu \mathrm{L}$ sample, followed by the incubation with $5 \mu \mathrm{L} 4 \mathrm{mM}$ DEVD-p-NA substrate at $37^{\circ} \mathrm{C}$ for $2 \mathrm{~h}$. The optical density values for the peroxidase reaction product were measured at $450 \mathrm{~nm}$ using a microplate reader.

Apoptosis assay. Cell apoptosis was assessed using Annexin V-FITC Apoptosis Detection Kit (\#14085, Abcam,) according to the manufacturer's instructions. Briefly, MPVECs were cultured in 6-well plates to reach 70-80\% confluency followed by the transfection and LPS stimulation as mentioned above. After $24 \mathrm{~h}$, cells were collected, centrifuged, and resuspended in $500 \mu \mathrm{L} 1 \times$ buffer solution at the concentration of $5 \times 10^{5}$ cells $/ \mathrm{mL}$. Then $5 \mu \mathrm{L}$ Annexin V-FITC and $5 \mu \mathrm{L}$ propidium iodide (PI) were added to the cells and incubated at room temperature for $5 \mathrm{~min}$ in the dark. The apoptosis ratio was quantified in the flow cytometry analysis using BD FACS software (BD Biosciences, San Jose, CA, USA).

Western blot. Total proteins $(40-80 \mu \mathrm{g})$ from MPVEC lysates were separated on an $8 \%$ SDS-PAGE gel under reducing conditions and then transferred to polyvinylidene fluoride membranes (MilliporeSigma, Burlington, MA, USA). After blocking, membranes were incubated with ROCK1 antibody (1:2000, \#ab45171, Abcam) and $\beta$-catenin antibody (1:2000, \#ab32572, Abcam) at $4{ }^{\circ} \mathrm{C}$ for $6 \mathrm{~h}$. After three washes with TBST buffer, membranes were incubated with goat anti-rabbit secondary antibody (1:3000, \# ab6721, Abcam) for 45 min. Protein bands were visualized using Alphalmager ${ }^{\mathrm{TM}} 2000$ Imaging System (Alpha Innotech, San Leandro, CA, USA) and the density of bands was quantified.

Statistical analysis. Data in this study are presented as mean \pm standard deviation. The statistical significance was analyzed using two-tailed Student's t-test or one-way ANOVA (SPSS software, version 24.0, Chicago, IL, USA). A value of $p<0.05$ was considered statistically significant. 
All experiments were performed in triplicate and repeated three times.

\section{Results}

MiR-539-5p was downregulated in mice with CLP-induced sepsis and the overexpression of miR-539-5p alleviated CLP-induced lung injury To study the regulatory role of miR-539-5p in sepsis-induced ALI, we established a mouse model of sepsis induced by CLP surgery. The induction of miR-539-5p expression in septic mice was achieved by intravenous injection of miR-539-5p agomir prior to CLP treatment. Compared to sham-operated group, the expression of miR-539-5p in the lung tissues was significantly inhibited following CLP surgery. The injection of miR-539-5p-expressing plasmid in septic mice significantly promoted the level of miR-539$5 \mathrm{p}$ compared to the ones administered with control miRNA (Fig. 1A). The degree of lung tissue damage was evaluated using lung injury scoring, in which miR-539-5p overexpression significantly improved the histological damage caused by CLP operation (Fig. 1B). As shown in the lung sections stained with $\mathrm{H} \& \mathrm{E}$, sham-operated group showed morphologically normal structure of pulmonary alveoli. CLP mice, on the contrary, showed thickened alveolar walls and septa, collapsed alveolar sacs, visible vascular congestion and hemorrhage. The histopathological features in septic mice injected with control agomir were similar to those in CLP group. The induction of miR-539-5p alleviated the pulmonary alveoli damage induced by sepsis (Fig. 1C). These results suggested that miR-539-5p protected mice from sepsis-induced lung injury following CLP surgery.

\section{Induction of miR-539-5p decreased apoptosis marker activity and proinflammatory cytokine production in the lung tissues of CLP mice}

We found that the transcriptional activity of caspase- 3 was significantly elevated in CLP-operated animals, whereas miR-539-5p overexpression induced a significant reduction of caspase- 3 activity in septic mice (Fig. 2A). The expressions of IL- $1 \beta$ and IL-6, two major proinflammatory cytokines, were significantly increased in septic animals at both mRNA and protein levels compared to sham-operated group. The induction of miR-539-5p in mice with ALI significantly suppressed the expressions of both cytokines (Fig. 2B-C). These results indicated that miR-539-5p ameliorated sepsis-induced apoptosis and proinflammatory cytokine production.

\section{Overexpression of miR-539-5p reduced LPS-induced apoptosis and inflammation in MPVECs}

In this study, we stimulated MPVECs with LPS to mimic pulmonary damage in vitro. LPS-treated cells demonstrated significantly lower level of miR-539-5p compared to untreated cells. Under the condition of LPS stimulation, wild-type MPVECs and the ones transfected with control mimics showed similar miR539-5p level, whereas the delivery of miR-539-5p mimics significantly increased the expression of miR-539-5p (Fig. 3A). The caspase-3 activity was remarkably promoted by LPS treatment, while miR539-5p overexpression resulted in a significant reduction of caspase-3 activity in LPS-treated MPVECs (Fig. 3B). LPS-induced secretion of IL- $1 \beta$ and IL-6 in MPVECs was also significantly impeded by miR539-5p (Fig. 3C-D). Flow cytometry data showed that LPS stimulated apoptosis in MPVECs, whereas the transfection of miR-539-5p mimics efficiently decreased the apoptosis ratio as compared to control mimics-transfected cells (Fig. 3E). The above findings suggested the anti-apoptotic and anti-inflammatory role of miR-539-5p in vitro.

\section{ROCK1 was directly targeted by miR-539-5p}

To explore the potential mechanisms underlying the involvement of miR-539-5p in ALI, we searched for the downstream target of miR-539-5p using bioinformatics analysis on TargetScan. ROCK1 was predicted as a direct target gene of miR-539-5p with a putative binding site (Fig. 4A). Dual-luciferase reporter assay showed that the luciferase activity of ROCK1-WT was significantly lower in cells transfected with miR-539-5p mimics compared to control mimics cells. In contrast, cells transfected with ROCK1-MUT reporter showed no difference on the luciferase activity between miR539-5p mimics and control mimics groups (Fig. 4B). Then, we measured the mRNA and protein levels of ROCK1 in wild-type and miR-539-5p-overexpressing MPVECs and found that the expression of ROCK1 was significantly downregulated by the induction of miR-539-5p (Fig. 4C).

\section{MiR-539-5p inhibited apoptosis and inflammatory responses via the suppression of ROCK1}

To further investigate whether miR-539-5p regulated apoptosis and inflammation via targeting ROCK1, we co-transfected MPVECs with miR-539-5p mimics and lentiviral vector expressing ROCK1 (or empty vector). The transfection efficacy was confirmed by significantly augmented expressions of ROCK1 at both mRNA and protein levels in MPVECs transfected with 


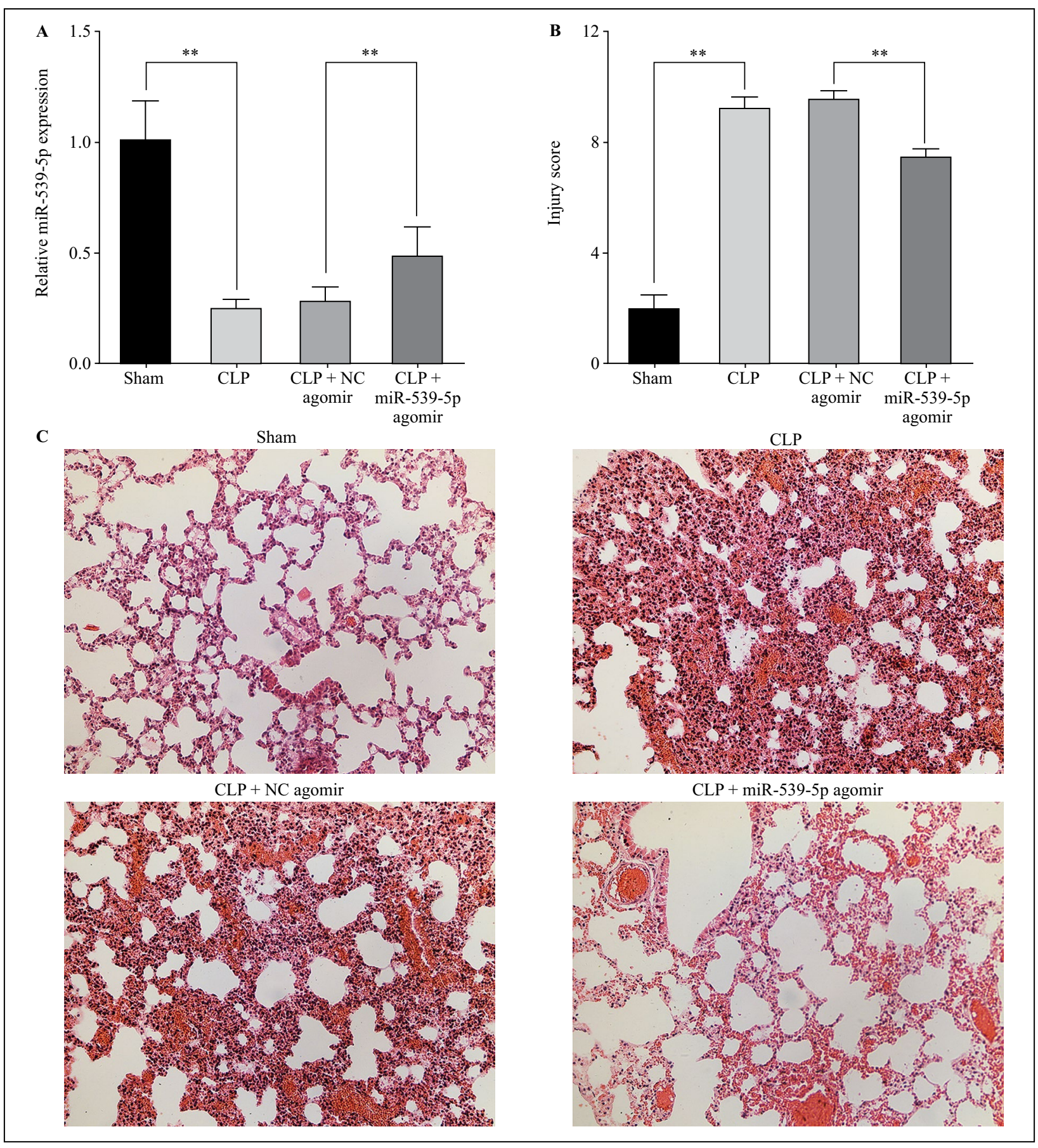

Figure 1. Pulmonary expression of miR-539-5p in the mouse model of CLP-induced sepsis and the effect of miR-539-5p overexpression on sepsis-induced lung injury. Adult male C57BL/6 mice were divided into four groups: CLP, CLP + NC agomir, CLP + miR-539-5p, and sham group ( $\mathrm{n}=6$ in each group). Animals in CLP, CLP + NC agomir, CLP + miR-539-5p groups were intravenously injected with vehicle, scrambled miRNA control, and miR-539-5p agomir, respectively, followed by CLP surgery. Sham group underwent the same surgical procedure of CLP without the ligation or puncture of the cecum. All animals were euthanized $24 \mathrm{~h}$ after the surgery, and their lung tissues were collected. A. Pulmonary expression of miR-539-5p was examined using qRT-PCR. B. Sectioned lung tissue samples were stained with H\&E and the degree of lung damage was determined using lung injury scoring. C. Representative histological images were shown $(400 \times$ magnification $)$. ${ }^{* *} \mathrm{p}<0.01$.

ROCK1 vector (Fig. 5A-B). We further explored the effect of ROCK1 overexpression on apoptosis and inflammation in MPVECs carrying miR-539-5p mimics.
Compared to control cells, MPVECs co-transfected with miR-539-5p and empty vector showed significantly impaired caspase- 3 activity. The induction of 

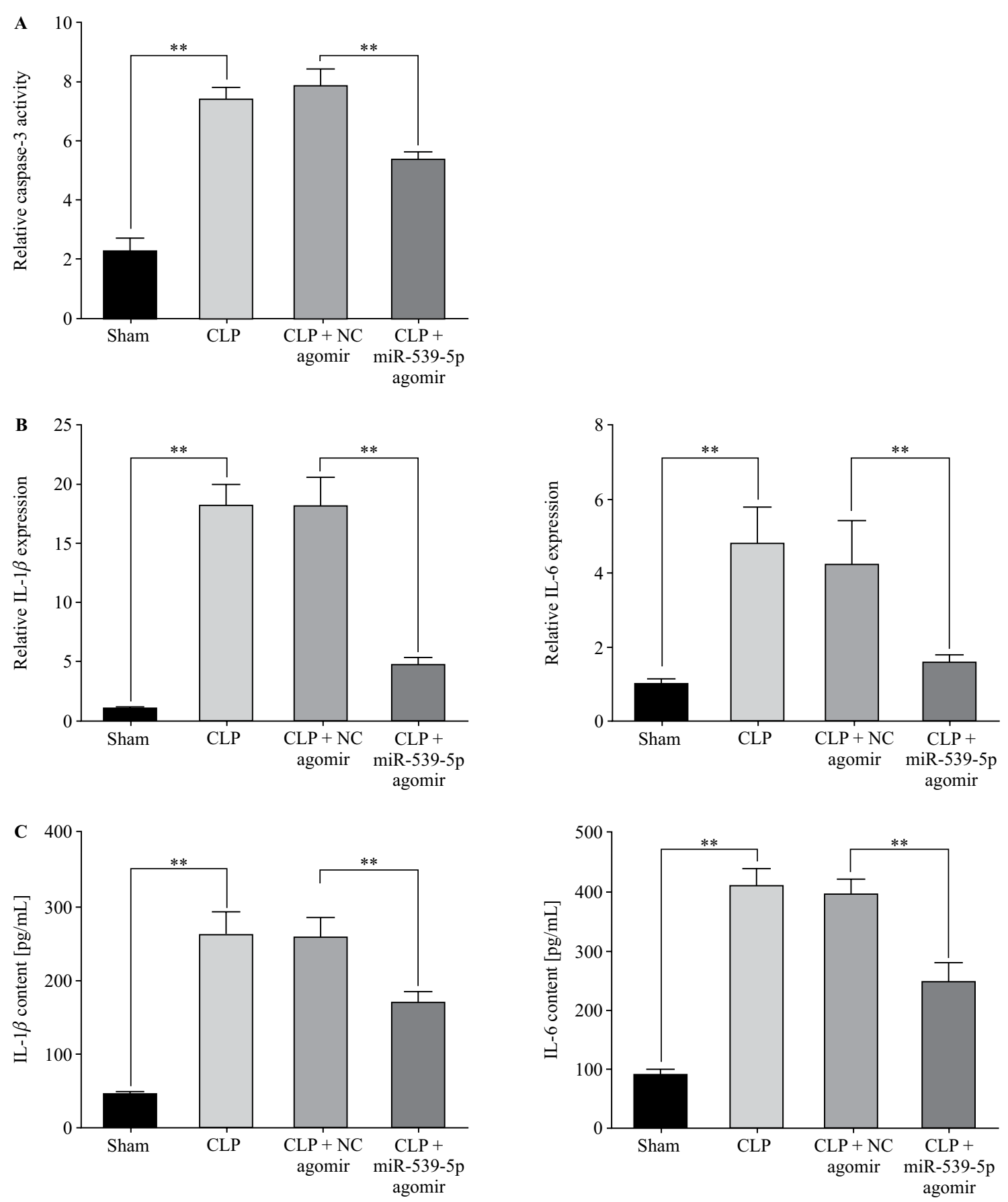

Figure 2. Effect of miR-539-5p overexpression on apoptosis and inflammation in mice lungs following CLP-induced sepsis. A. The transcriptional activity of caspase-3 in lung tissues was measured using Caspase-3 Assay Kit. B-C. The pulmonary mRNA and protein levels of IL- $1 \beta$ and IL- 6 were examined using qRT-PCR and ELISA, respectively. ${ }^{* *}$ p $<0.01$.

ROCK1, however, increased the activity of caspase-3 back to the level that was shown in control group (Fig. 5C). The production of proinflammatory cytokines in MPVECs was largely inhibited by miR-539-5p overexpression, whereas ROCK1 led to a significant upregulation of IL- $1 \beta$ and IL- 6 at both mRNA and protein levels (Fig. 5D-E). Moreover, the overexpression of miR-539-5p significantly decreased the apoptosis ratio of MPVECs, whereas the transfection of ROCK1-expressing vectors substantially facilitated MPVECs apoptosis (Fig. 5F). Taken together, these data indicated that miR-539-5p inhibited apoptosis 

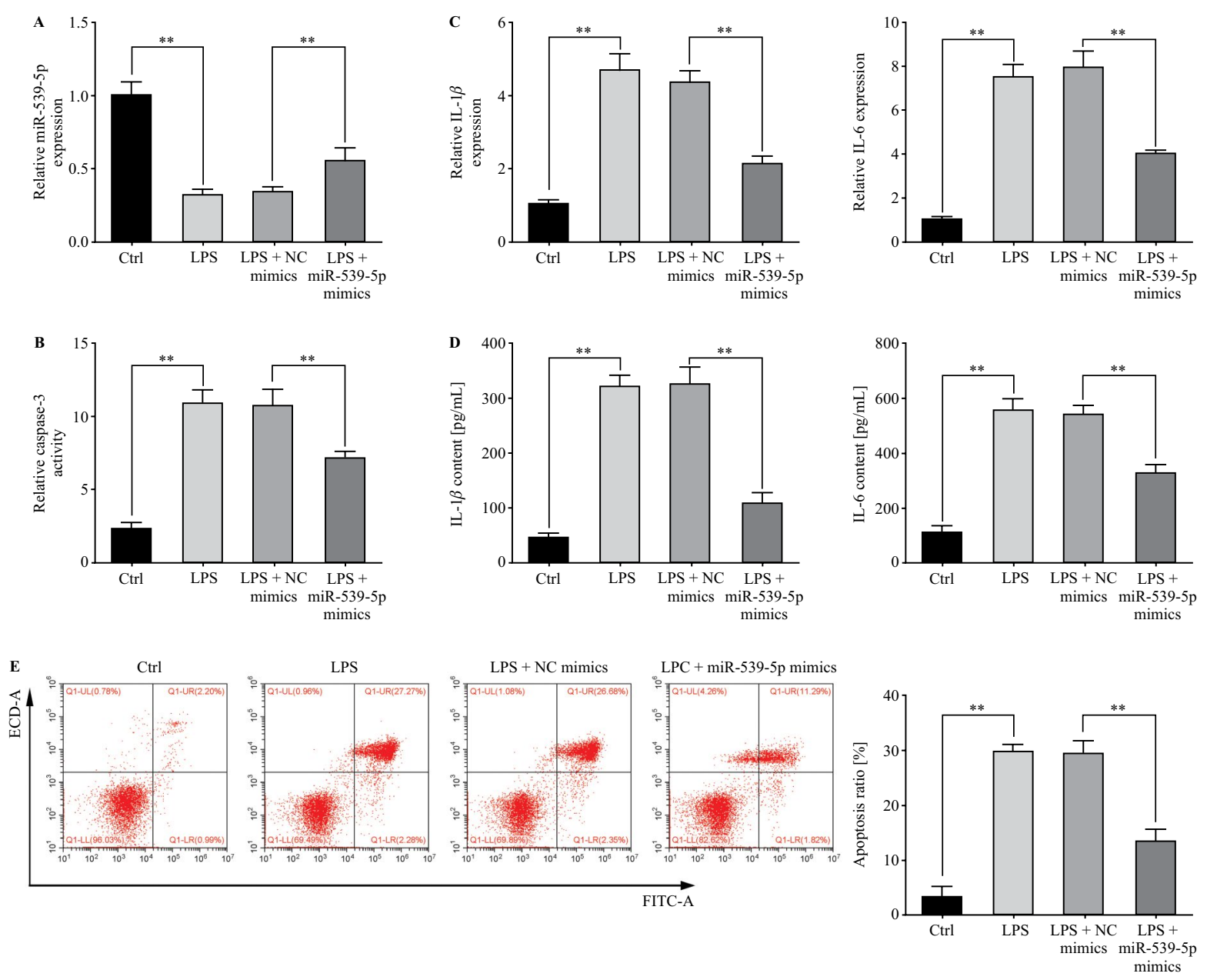

Figure 3. Induction of miR-539-5p affects the expression of apoptosis marker and proinflammatory cytokines in LPS-stimulated primary murine pulmonary microvascular endothelial cells (MPVECs). Isolated MPVECs were divided into four groups: LPS (lipopolysaccharide), LPS + NC mimics, LPS + miR-539-5p mimics and Ctrl. Cells in LPS + NC mimics and LPS + miR-539-5p mimics groups were transfected with control mimics and miR-539-5p mimics, respectively, followed by the stimulation with $1 \mathrm{mg} / \mathrm{mL}$. Cells in LPS group were stimulated with LPS without transfection. Control (Ctrl) cells remained untreated. A. Relative expression of miR-539-5p was assessed using qRT-PCR $6 \mathrm{~h}$ after the stimulation with lipopolysaccharides (LPS). B. The transcriptional activity of caspase-3 was examined 30 min following the treatment. C-D. The mRNA and protein levels of IL- $1 \beta$ and IL- 6 were examined using qRT-PCR and ELISA, respectively. E. The apoptosis ratio in transfected MPVECs was calculated using flow cytometry analysis. ${ }^{*} \mathrm{p}<0.01$.

and inflammation in sepsis-induced ALI via the suppression of its target gene ROCK1.

\section{Discussion}

Sepsis is a leading etiology of ALI that leads to more severe illness and higher mortality rates compared to non-sepsis risk factors [22]. As a syndrome developed from excessive inflammation, ALI affords multiple potential therapeutic targets and signaling pathways [23]. In the present study, we reported for the first time that miR-539-5p mitigated the development of sepsis-induced ALI both in vivo and in vitro. The induction of miR-539-5p in septic models decreased the expression of proinflammatory cytokines, IL- $1 \beta$ and IL-6, and suppressed the transcriptional activity of apoptosis marker, caspase-3. Further investigation revealed that miR-539-5p reduced apoptosis and inflammation in sepsis-induced ALI via the negative regulation of its downstream target ROCK1.

Mouse CLP model closely resembles the pathophysiology of human sepsis caused by abdominal perforations and has been extensively used in the research of sepsis-induced organ dysfunction [24]. In 
$\mathbf{A}$

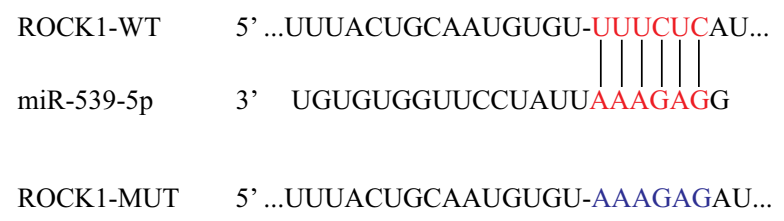

B

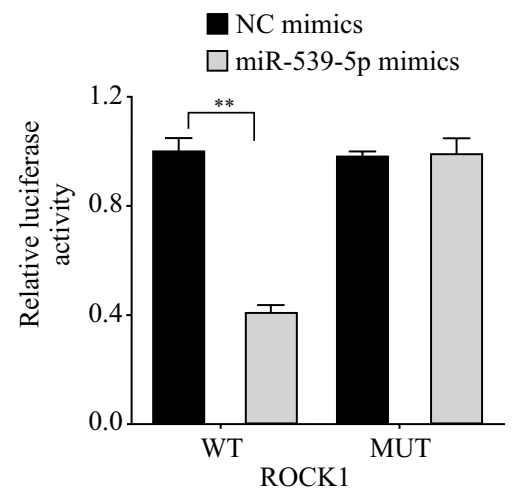

C

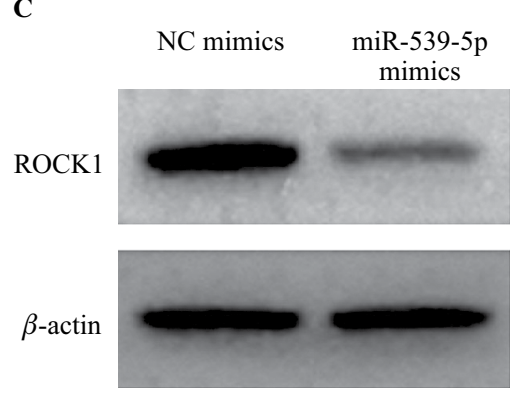

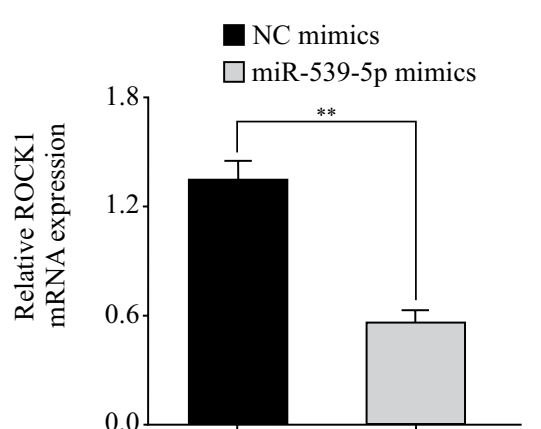

Figure 4. ROCK1 is a direct target of miR-539-5p. ROCK1 was predicted as a direct downstream target of miR-539-5p using TargetScan. A. The putative ROCK1 binding site for miR-539-5p (ROCK1-WT) and the designed mutant sequence (ROCK1-MUT) were shown. B. HEK-293T cells were co-transfected with NC mimics (or miR-539-5p mimics) and ROCK1WT (or ROCK1-MUT). The luciferase activity was measuring using dual-luciferase reporter assay. C. The expression of ROCK1 in MPVECs transfected with NC mimics and miR-539-5p mimics were examined using Western blot and qRT-PCR.

this study, we successfully established the mouse model of sepsis-induced ALI by performing CLP surgery and showed morphological abnormalities in the lung, including thickened alveolar walls, collapsed alveolar sacs, vascular congestion and hemorrhage in CLP mice as compared to sham-operated group. Enhanced cell apoptosis and the induction of proinflammatory mediators during sepsis play crucial roles in the pathogenesis of ALI [25]. IL- $1 \beta$ and IL-6 are two proinflammatory cytokines actively secreted in response to the inflammatory cascade in ALI $[26,27]$. The inhibition of apoptotic signaling and inflammatory responses might potentially ameliorate sepsis-induced lung damage. The caspase- 3 activity is an important marker of cellular apoptosis. Increased activation of caspase-3 has been reported in the animal models of ALI [28, 29 ]. In the current study, enhanced caspase- 3 activity, elevated production of IL- $1 \beta$ and IL-6, together with the increased apoptosis ratio was observed following CLP operation. LPS is an endotoxin constituting the outer membrane of Gram-negative bacteria, and has been widely used to induce acute lung injury in research [30]. In the in vitro model, incubation with LPS led to a significant induction of inflammatory cytokine levels and apoptosis in MPVECs compared to untreated cells.

The potential involvement of miRNAs in ALI was first shown in a mouse model of LPS-induced lung injury, in which certain types of miRNAs were significantly downregulated and the others were remarkably upregulated or remained unaltered [31]. Further investigations on the role of miRNAs in ALI suggested that miRNAs might regulate the inflammatory and apoptotic pathways during ALI progression by targeting specific molecules or regulating downstream genes [32]. Tuerdi et al. reported that the downregulation of miR-155 inhibited lung apoptosis and inflammation, and increased the survival rate in CLP-induced ALI mice by targeting SIRT1 [33]. The depletion of miR1246 reduced apoptosis, the release of IL- $1 \beta$, and neutrophil infiltration in mice with ALI through the repression of its downstream target ACE2 [34]. Xie et al. showed significantly reduced expression of miR127 during lung injury in vivo, while the administration of miR-127 probes attenuated pulmonary inflammation through the regulation of CD46 in macrophages [35]. In another animal study, the upregulation of miR146a suppressed the secretion of TNF- $\alpha$, IL-6, 

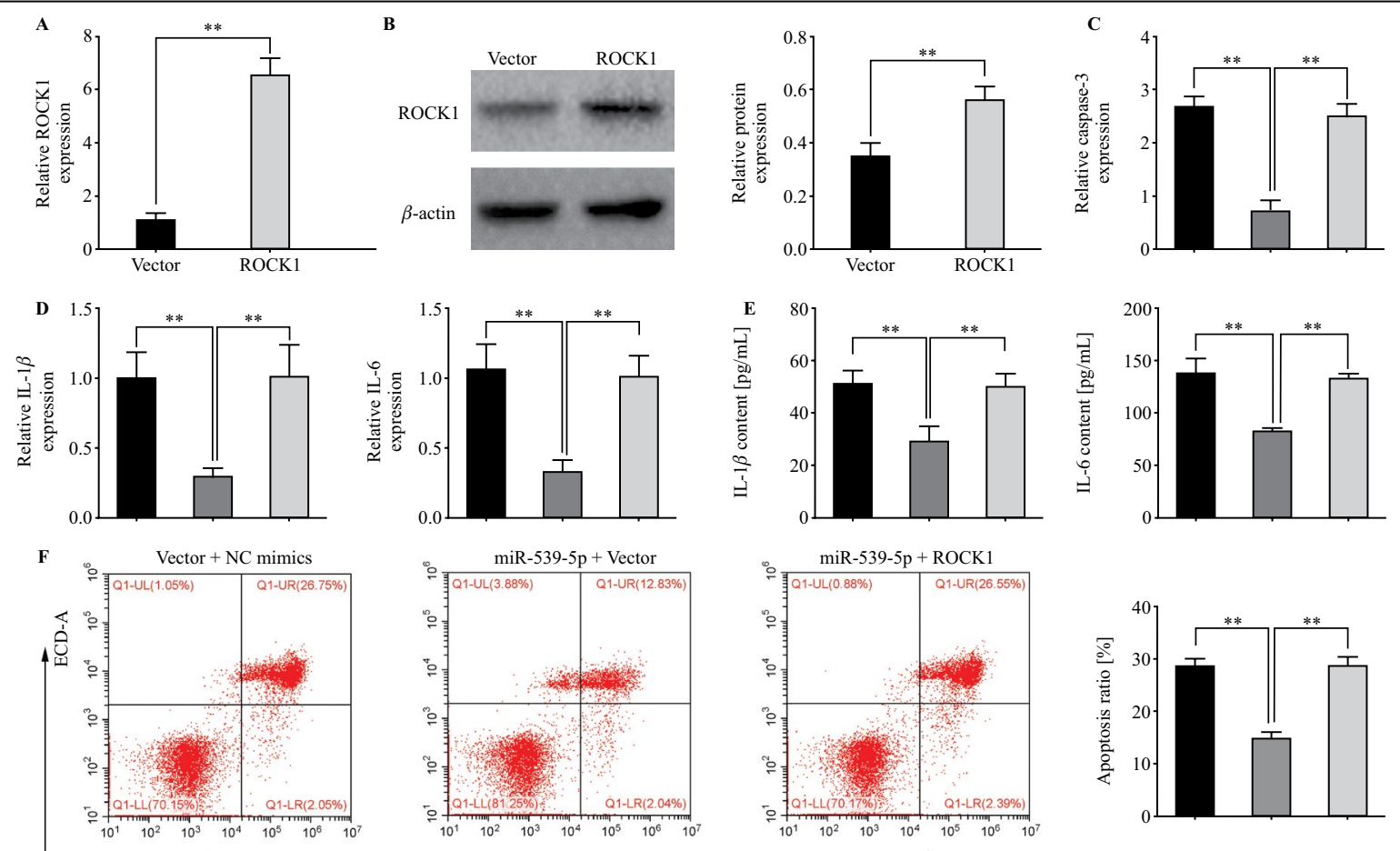

Vector $+\mathrm{NC}$ mimics

$\square$ miR-539-5p + ROCK1

Figure 5. MiR-539-5p mediated apoptosis and inflammation via regulating ROCK1. MPVECs were divided into three groups: miR-539-5p + Vector, miR-539-5p + ROCK1 and Vector + NC mimics. In miR-539-5p + Vector and miR-539$5 p+$ ROCK1 groups, MPVECs were co-transfected with miR-539-5p mimics and lentiviral vector expressing ROCK1 (or empty lentiviral vector) $24 \mathrm{~h}$ before LPS stimulation. MPVECs in Vector + NC mimics group were co-transfected with empty lentiviral vector and control mimics. A-B. To evaluate the transfection efficacy, the mRNA and protein levels of ROCK1 in miR-539-5p + Vector and miR-539-5p + ROCK1 groups were analyzed using qRT-PCR and Western blot. C. The transcriptional activity of caspase- 3 was examined $30 \mathrm{~min}$ post-stimulation. D-E. The mRNA and protein levels of IL- $1 \beta$ and IL-6 were determined using qRT-PCR and ELISA, respectively. F. The apoptosis ratio in transfected MPVECs was calculated using flow cytometry analysis. $* * \mathrm{p}<0.01$.

and IL-1 $\beta$ in LPS-induced ALI model via the inhibition of IRAK-1 and TRAF-6 [36]. In our study, we showed that the level of miR-539-5p was significantly downregulated in CLP-operated mice compared to the sham group. The delivery of miR-539-5p mimics prior to CLP surgery efficiently alleviated sepsis-induced pulmonary injury, reduced caspase- 3 activity and apoptosis, suppressed the induction of inflammatory cytokines IL- $1 \beta$ and IL- 6 . The anti-apoptotic and anti-inflammatory effect of miR-539-5p were also shown in LPS-treated MPVECs.

ROCK1 has been predicted and confirmed as a direct downstream target of miR-539-5p in our study. A previous study in CLP rat model showed that the activation of ROCK1 was involved in the pathogenesis of sepsis-induced ALI with a potential mechanism related to oxidative stress and apoptosis [37]. Here, by enhancing the expression of ROCK1 in MPVECs, the beneficial effect of miR-539-5p in ALI-associated apoptosis and inflammation was significantly reversed. It indicated that miR-539-5p ameliorated sepsis-induced ALI by repressing its downstream target ROCK1.

In conclusion, miR-539-5p alleviated sepsis-induced apoptosis and proinflammatory cytokine production during ALI by downregulating the expression of ROCK1. This study highlighted the potential of miR-539-5p as a therapeutic target for the management of sepsis-induced ALI. 


\section{Competing interests}

The authors declare that they have no competing interests, and all authors should confirm its accuracy.

\section{Availability of data and materials}

All data generated or analyzed during this study are included in this published article.

\section{Authors' contributions}

LM and CHW conceived and designed the experiments, HHC analyzed and interpreted the results of the experiments, LTJ performed the experiments.

\section{Ethics approval and consent to participate}

The animal use protocol listed below has been reviewed and approved by the Animal Ethical and Welfare Committee of Hubei Provincial Hospital.

\section{Patient consent for publication}

Not Applicable.

\section{Informed consent}

Written informed consent was obtained from a legally authorized representative(s) for anonymized patient information to be published in this article.

\section{References}

1. Sagy M, Al-Qaqaa Y, Kim P. Definitions and pathophysiology of sepsis. Curr Probl Pediatr Adolesc Health Care. 2013; 43(10): 260-263, doi: 10.1016/j.cppeds.2013.10.001, indexed in Pubmed: 24295606.

2. Gando S. Microvascular thrombosis and multiple organ dysfunction syndrome. Crit Care Med. 2010; 38(2 Suppl): S35-S42, doi: 10.1097/CCM.0b013e3181c9e31d, indexed in Pubmed: 20083912.

3. Levitt JE, Matthay MA. The utility of clinical predictors of acute lung injury: towards prevention and earlier recognition. Expert Rev Respir Med. 2010; 4(6): 785-797, doi: 10.1586/ ers.10.78, indexed in Pubmed: 21128753.

4. Johnson ER, Matthay MA. Acute lung injury: epidemiology, pathogenesis, and treatment. J Aerosol Med Pulm Drug Deliv. 2010; 23(4): 243-252, doi: 10.1089/jamp.2009.0775, indexed in Pubmed: 20073554.

5. Levitt JE, Matthay MA. Clinical review: Early treatment of acute lung injury--paradigm shift toward prevention and treatment prior to respiratory failure. Crit Care. 2012; 16(3): 223, doi: 10.1186/cc11144, indexed in Pubmed: 22713281.

6. Park WY, Goodman RB, Steinberg KP, et al. Cytokine balance in the lungs of patients with acute respiratory distress syndrome. Am J Respir Crit Care Med. 2001; 164(10 Pt 1): 1896-1903, doi: 10.1164/ajrccm.164.10.2104013, indexed in Pubmed: 11734443.
7. Goodman RB, Strieter RM, Martin DP, et al. Inflammatory cytokines in patients with persistence of the acute respiratory distress syndrome. Am J Respir Crit Care Med. 1996; 154(3 Pt 1): 602-611, doi: 10.1164/ajrccm.154.3.8810593, indexed in Pubmed: 8810593.

8. Martin TR, Nakamura M, Matute-Bello G. The role of apoptosis in acute lung injury. Crit Care Med. 2003; 31(4 Suppl): S184-S188, doi: 10.1097/01.CCM.0000057841.33876.B1, indexed in Pubmed: 12682438.

9. Perl M, Chung CS, Perl U, et al. Fas-induced pulmonary apoptosis and inflammation during indirect acute lung injury. Am J Respir Crit Care Med. 2007; 176(6): 591-601, doi: 10.1164/rccm.200611-1743OC, indexed in Pubmed: 17600273.

10. Kitamura Y, Hashimoto S, Mizuta N, et al. Fas/FasL-dependent apoptosis of alveolar cells after lipopolysaccharide-induced lung injury in mice. Am J Respir Crit Care Med. 2001; 163(3 Pt 1): 762-769, doi: 10.1164/ajrccm.163.3.2003065, indexed in Pubmed: 11254536.

11. Kingsley SM, Bhat BV. Role of microRNAs in sepsis. Inflamm Res. 2017; 66(7): 553-569, doi: 10.1007/s00011-017-1031-9, indexed in Pubmed: 28258291.

12. Adameova AD, Bhullar SK, Elimban V, et al. Activation of -adrenoceptors may not be involved in arrhythmogenesis in ischemic heart disease. Rev Cardiovasc Med. 2018; 19(3): 97-101, doi: 10.31083/j.rcm.2018.03.3181, indexed in Pubmed: 31054558.

13. Yang Q, Zhang D, Li Ya, et al. Paclitaxel alleviated liver injury of septic mice by alleviating inflammatory response via microRNA-27a/TAB3/NF- B signaling pathway. Biomed Pharmacother. 2018; 97: 1424-1433, doi: 10.1016/j.biopha.2017.11.003, indexed in Pubmed: 29156532.

14. Feng Y, Wang J, Yuan Y, et al. miR-539-5p inhibits experimental choroidal neovascularization by targeting CXCR7. FASEB J. 2018; 32(3): 1626-1639, doi: 10.1096/fj.201700640R, indexed in Pubmed: 29146732.

15. Hu L, Liu Y, Wang B, et al. MiR-539-5p negatively regulates migration of rMSCs induced by Bushen Huoxue decoction through targeting Wnt5a. Int J Med Sci. 2019; 16(7): 998-1006, doi: 10.7150/ijms.33437, indexed in Pubmed: 31341413.

16. National Research Council Institute for Laboratory Animal R. Guide for the Care and Use of Laboratory Animals. Washington (DC): National Academies Press (US), 1996.

17. Matsuda N, Hattori Y, Jesmin S, et al. Nuclear factor-kappaB decoy oligodeoxynucleotides prevent acute lung injury in mice with cecal ligation and puncture-induced sepsis. Mol Pharmacol. 2005; 67(4): 1018-1025, doi: 10.1124/mol.104.005926, indexed in Pubmed: 15576632.

18. Ruiz S, Vardon-Bounes F, Merlet-Dupuy V, et al. Sepsis modeling in mice: ligation length is a major severity factor in cecal ligation and puncture. Intensive Care Med Exp. 2016; 4(1): 22, doi: 10.1186/s40635-016-0096-z, indexed in Pubmed: 27430881.

19. Morishita Y, Imai T, Yoshizawa H, et al. Delivery of microRNA-146a with polyethylenimine nanoparticles inhibits renal fibrosis in vivo. Int J Nanomedicine. 2015; 10: 3475-3488, doi: 10.2147/IJN.S82587, indexed in Pubmed: 25999712.

20. Matute-Bello G, Downey G, Moore BB, et al. Acute Lung Injury in Animals Study Group. An official American Thoracic Society workshop report: features and measurements of experimental acute lung injury in animals. Am J Respir Cell Mol Biol. 2011; 44(5): 725-738, doi: 10.1165/rcmb.20090210ST, indexed in Pubmed: 21531958.

21. Hebbel RP, Vercellotti GM, Pace BS, et al. The HDAC inhibitors trichostatin $\mathrm{A}$ and suberoylanilide hydroxamic 
acid exhibit multiple modalities of benefit for the vascular pathobiology of sickle transgenic mice. Blood. 2010;115(12): 2483-2490, doi: 10.1182/blood-2009-02-204990, indexed in Pubmed: 20053759.

22. Sevransky JE, Martin GS, Shanholtz C, et al. Mortality in sepsis versus non-sepsis induced acute lung injury. Crit Care. 2009; 13(5): R150, doi: 10.1186/cc8048, indexed in Pubmed: 19758459.

23. Sweeney RM, Griffiths M, McAuley D. Treatment of acute lung injury: current and emerging pharmacological therapies. Semin Respir Crit Care Med. 2013; 34(4): 487-498, doi: 10.1055/s-0033-1351119, indexed in Pubmed: 23934717.

24. Rittirsch D, Hoesel LM, Ward PA. The disconnect between animal models of sepsis and human sepsis. J Leukoc Biol. 2007; 81(1): 137-143, doi: 10.1189/jlb.0806542, indexed in Pubmed: 17020929.

25. Chopra M, Reuben JS, Sharma AC. Acute lung injury:apoptosis and signaling mechanisms. Exp Biol Med (Maywood). 2009; 234(4): 361-371, doi: 10.3181/0811-MR-318, indexed in Pubmed: 19176873.

26. Ganter MT, Roux J, Miyazawa B, et al. Interleukin-1beta causes acute lung injury via alphavbeta5 and alphavbeta6 integrin-dependent mechanisms. Circ Res. 2008; 102(7): 804-812, doi: 10.1161/CIRCRESAHA.107.161067, indexed in Pubmed: 18276918.

27. Cross LJ, Matthay MA. Biomarkers in acute lung injury: insights into the pathogenesis of acute lung injury. Crit Care Clin. 2011; 27(2): 355-377, doi: 10.1016/j.ccc.2010.12.005, indexed in Pubmed: 21440206.

28. Fielhaber JA, Carroll SF, Dydensborg AB, et al. Inhibition of mammalian target of rapamycin augments lipopolysaccharide-induced lung injury and apoptosis. J Immunol. 2012; 188(9): 4535-4542, doi: 10.4049/jimmunol.1003655, indexed in Pubmed: 22450807.

29. Wang L, Ye Y, Su HB, et al. The anesthetic agent sevoflurane attenuates pulmonary acute lung injury by modulating apop- totic pathways. Braz J Med Biol Res. 2017; 50(3): e5747, doi: 10.1590/1414-431X20165747, indexed in Pubmed: 28225890.

30. Jeyaseelan S, Chu HW, Young SK, et al. Transcriptional profiling of lipopolysaccharide-induced acute lung injury. Infect Immun. 2004; 72(12): 7247-7256, doi: 10.1128/IAI.72.12.72477256.2004, indexed in Pubmed: 15557650.

31. Cai ZG, Zhang SM, Zhang Y, et al. MicroRNAs are dynamically regulated and play an important role in LPS-induced lung injury. Can J Physiol Pharmacol. 2012; 90(1): 37-43, doi: 10.1139/y11-095, indexed in Pubmed: 22185353.

32. Cao Y, Lyu YI, Tang J, et al. MicroRNAs: Novel regulatory molecules in acute lung injury/acute respiratory distress syndrome. Biomed Rep. 2016; 4(5): 523-527, doi: 10.3892/ br.2016.620, indexed in Pubmed: 27123242.

33. Liu Y, Guan H, Zhang JL, et al. Acute downregulation of miR-199a attenuates sepsis-induced acute lung injury by targeting SIRT1. Am J Physiol Cell Physiol. 2018; 314(4): C449-C455, doi: 10.1152/ajpcell.00173.2017, indexed in Pubmed: 29351405.

34. Fang Y, Gao F, Hao J, et al. microRNA-1246 mediates lipopolysaccharide-induced pulmonary endothelial cell apoptosis and acute lung injury by targeting angiotensin-converting enzyme 2. Am J Transl Res. 2017; 9(3): 1287-1296, indexed in Pubmed: 28386354.

35. Xie T, Liang J, Liu N, et al. MicroRNA-127 inhibits lung inflammation by targeting IgG Fc $\gamma$ receptor I. J Immunol. 2012; 188(5): 2437-2444, doi: 10.4049/jimmunol.1101070, indexed in Pubmed: 22287715.

36. Zeng Z, Gong H, Li Y, et al. Upregulation of miR-146a contributes to the suppression of inflammatory responses in LPS-induced acute lung injury. Exp Lung Res. 2013; 39(7): 275-282, doi: 10.3109/01902148.2013.808285, indexed in Pubmed: 23848342.

37. Cinel I, Ark M, Dellinger $P$, et al. Involvement of Rho kinase (ROCK) in sepsis-induced acute lung injury. J Thorac Dis. 2012; 4(1): 30-39, doi: 10.3978/j.issn.2072-1439.2010.08.04, indexed in Pubmed: 22295165.

Submitted: 27 September, 2019

Accepted after reviews: 14 November, 2019 Available as AoP: 11 December, 2019 